Check for updates

Cite this: J. Mater. Chem. A, 2019, 7, 16109

Received 25th May 2019

Accepted 21st June 2019

DOI: $10.1039 / c 9 t a 05554 a$

rsc.li/materials-a

\section{Layered metal vanadates with different interlayer cations for high-rate $\mathrm{Na}$-ion storage $\uparrow$}

\author{
Haijian Huang, (D) a Tian Tian, (D) ${ }^{\mathrm{b}}$ Long Pan, (D) ${ }^{\mathrm{a}}$ Xi Chen, ${ }^{\mathrm{a}}$ Elena Tervoort, ${ }^{\mathrm{a}}$ \\ Chih-Jen Shih (iD ${ }^{b}$ and Markus Niederberger (iD *a
}

\begin{abstract}
Layer-structured metal vanadates have been regarded as promising candidates for high-rate $\mathrm{Na}$-ion storage. However, without a detailed understanding of the relationship between the interlayer metal ions and the cycling performance, it remains a difficult task to systematically explore layered metal vanadates as high performance electrode materials. Herein, a series of metal vanadates with different interlayer cations such as $\mathrm{Co}^{2+}$ and $\mathrm{Zn}^{2+}$ are prepared and applied as $\mathrm{Na-ion}$ battery anodes. First principles simulations and ex situ X-ray diffraction measurements demonstrate that the $\mathrm{Na}$-ion storage performance of the layered metal vanadates is closely related to the structural stress induced by $\mathrm{Na}^{+}$insertion, and the ion diffusion barrier, as well as the structural reversibility. In addition, a double-interlayer-cation metal vanadate, i.e., $\mathrm{Co}_{0.16} \mathrm{Zn}_{0.09} \mathrm{~V}_{2} \mathrm{O}_{5} \cdot n \mathrm{H}_{2} \mathrm{O}$, is reported for the first time as a high-rate $\mathrm{Na}$-ion battery anode. This compound successfully combines the favorable features of $\mathrm{Co}_{0.25} \mathrm{~V}_{2} \mathrm{O}_{5} \cdot n \mathrm{H}_{2} \mathrm{O}$ and $\mathrm{Zn}_{0.25} \mathrm{~V}_{2^{-}}$ $\mathrm{O}_{5} \cdot n \mathrm{H}_{2} \mathrm{O}$, resulting in the best cycling performance. $\mathrm{CV}$ analysis and operando $\mathrm{X}$-ray diffraction measurements reveal a large pseudocapacitive contribution and small volume change of $\mathrm{CO}_{0.16} \mathrm{Zn}_{0.09} \mathrm{~V}_{2} \mathrm{O}_{5}$ - $n \mathrm{H}_{2} \mathrm{O}$ during cycling. Our study presents a versatile concept for the optimization of metal vanadates for $\mathrm{Na}$-ion storage, which may open a promising direction for developing high-rate energy storage materials.
\end{abstract}

\section{Introduction}

Na-ion batteries (NIBs) have been in the spotlight for 30 years as promising candidates for large-scale energy storage, mainly because of the abundance and low cost of sodium resources. ${ }^{1-6}$ However, the sluggish ion diffusion kinetics as a result of the

${ }^{a}$ Department of Materials, Laboratory for Multifunctional Materials, ETH Zürich, Vladimir-Prelog-Weg 5, 8093 Zürich, Switzerland. E-mail: markus.niederberger@ mat.ethz.ch

${ }^{b}$ Department of Chemistry and Applied Biosciences, Institute for Chemical and Bioengineering, ETH Zürich, Vladimir-Prelog-Weg 1, Zürich, 8093, Switzerland

$\uparrow$ Electronic supplementary information (ESI) available: Experimental section, Table S1, and Fig. S1-S14. See DOI: 10.1039/c9ta05554a large ionic radius of sodium remained a major difficulty for developing electrode materials with not only high energy density but also decent rate capability, which is the key to highperformance NIBs. ${ }^{7-9}$ To explore high-rate NIB electrode materials, tremendous efforts have been devoted to minimizing the particle size $\mathrm{e}^{\mathbf{1 0 - 1 2}}$ and manipulating the particle shape, such as creating hollow or yolk-shell structures. ${ }^{13-15}$ Despite the fact that nanoscaling and nanostructuring can efficiently improve the rate capability, it usually results in complicated and expensive synthesis pathways. Besides, nanometer-sized or hollow-structured electrode materials inherently suffer from poor volumetric energy density. Thus, exploring new pathways and approaches to high-rate Na-ion storage materials is a timely and highly relevant endeavour to improve the electrochemical performance of NIBs.

Vanadium-based materials are a particularly promising type of materials for high-performance Na-ion storage. ${ }^{16-18}$ Among them, layered metal vanadates have been proved to show intercalation pseudocapacitive behaviors due to their large open channels within the framework of the crystal structure, which offer spacious pathways for the insertion and extraction of the alkali metal ions. ${ }^{19-26}$ Besides, they show small volume changes during $\mathrm{Na}^{+}$insertion ${ }^{27-29}$ and high rate capability even when the particle size is at the level of micrometers, which is especially beneficial for practical applications..$^{27,28,30}$ In a previous report, we showed that the fast Na-ion diffusion in zinc vanadate leads to high-rate Na-ion storage capability. ${ }^{27}$ In addition, cation doping or substitution are promising strategies to further enhance the electrochemical performances for Na-ion storage. ${ }^{31-33}$ However, up to now, the study of the relationship between the interlayer cations of the metal vanadates and their Na-ion storage performance remained nearly unexplored, although it is of great importance for rationally designing highperformance layered metal vanadates for high-rate NIBs.

We prepared a series of micron-sized layered metal vanadates $\left(\mathrm{Zn}_{0.25} \mathrm{~V}_{2} \mathrm{O}_{5} \cdot n \mathrm{H}_{2} \mathrm{O}(\mathrm{ZVO}), \mathrm{Co}_{0.25} \mathrm{~V}_{2} \mathrm{O}_{5} \cdot n \mathrm{H} 2 \mathrm{O}(\mathrm{CVO})\right.$, and $\mathrm{Co}_{0.16} \mathrm{Zn}_{0.09} \mathrm{~V}_{2} \mathrm{O}_{5} \cdot n \mathrm{H}_{2} \mathrm{O}$ (CZVO)) and demonstrate their high electrochemical performance as NIB anodes. To study the Na- 
ion storage mechanism and the optimization effect of the interlayer cations, density functional theory (DFT) calculations and ex situ X-ray diffraction (XRD) measurements were performed. The results clearly indicate that the Na-ion storage capability and cycling stability of the layered metal vanadates are closely related to the interlayer metal ions. The study of metal vanadates with two interlayer cations is interesting, because it allows to combine the advantageous properties of different metal ions. Taking advantage of the high charge storage capability of CVO and the high structural stability of $\mathrm{ZVO}$, the double-interlayer-cation metal vanadate $\mathrm{Co}_{0.16} \mathrm{Zn}_{0.09^{-}}$ $\mathrm{V}_{2} \mathrm{O}_{5} \cdot n \mathrm{H}_{2} \mathrm{O}$ (CZVO) prepared in this study turns out to exhibit the best cycling performance with $85 \mathrm{~mA} \mathrm{~h} \mathrm{~g}^{-1}$ obtained after 1000 cycles at a high current density of $3 \mathrm{~A} \mathrm{~g}^{-1}$. Furthermore, CV analysis demonstrates the dominant pseudocapacitive behavior in CZVO. Operando XRD measurements also reveal the high structural reversibility and negligible volume change of CZVO during $\mathrm{Na}^{+}$insertion/extraction. Thus, beyond the optimization of particle size and shape, this study provides a new approach, i.e., tuning the interlayer cations of the layered metal vanadates, to efficiently enhance the electrochemical performance of electrode materials for high-rate Na-ion storage.

\section{Results and discussion}

Powder XRD patterns of the as-prepared metal vanadates are shown in Fig. 1. All samples exhibit intense diffraction peaks typical for highly crystalline compounds. The XRD patterns of
ZVO (Fig. 1a) and CVO (Fig. 1d) can be indexed to pure $\mathrm{Zn}_{0.25^{-}}$ $\mathrm{V}_{2} \mathrm{O}_{5} \cdot n \mathrm{H}_{2} \mathrm{O}$ (ICSD PDF no. 04-012-3619) ${ }^{34}$ and $\mathrm{Co}_{0.25} \mathrm{~V}_{2} \mathrm{O}_{5} \cdot n \mathrm{H}_{2} \mathrm{O}$ (ICSD PDF no. 04-011-1841), ${ }^{35}$ respectively. In addition, the peaks for both samples are dominated by (001) reflections due to their highly anisotropic shape as discussed below. As shown in Fig. S1 and $\mathrm{S} 2, \uparrow$ both ZVO and CVO possess layered structures with $\mathrm{MO}_{6}(\mathrm{M}=\mathrm{Zn}, \mathrm{Co})$ octahedra acting as pillars between the $\mathrm{VO}_{x}$ layers. ${ }^{34,35}$ The open framework of the crystal structures is able to provide spacious pathways for fast solid-state ion diffusion, which is especially beneficial for high-rate alkali-ion storage. Fig. 1g presents the XRD result for CZVO, which exhibits a comparable diffraction pattern. This observation clearly indicates that CZVO has a similar crystal structure like ZVO and CVO. According to XRD, the interlayer distances of ZVO, CVO and CZVO are determined to be $10.14 \AA$ А, $10.06 \AA$ and $10.39 \AA$, respectively. The morphology of the samples was explored by scanning electron microscopy (SEM) and transmission electron microscopy (TEM). The SEM images at high magnification (Fig. 1b, e and h) and low magnification (Fig. S3†) as well as the TEM images (Fig. S4 $\dagger$ ) reveal the highly anisotropic, 1-dimensional morphology with smooth surfaces and shapes of straight edges for all samples. The structures are nearly transparent to the electron beam, pointing to a thin beltlike shape. The belts of the three samples are all tens of micrometers long and hundreds of nanometers wide. In Fig. 1h, an occasionally found twisted CZVO belt reveals a width to thickness ratio of $\sim 6.2: 1$, further confirming the belt-like morphology of the sample. Although the initial Co to $\mathrm{Zn}$
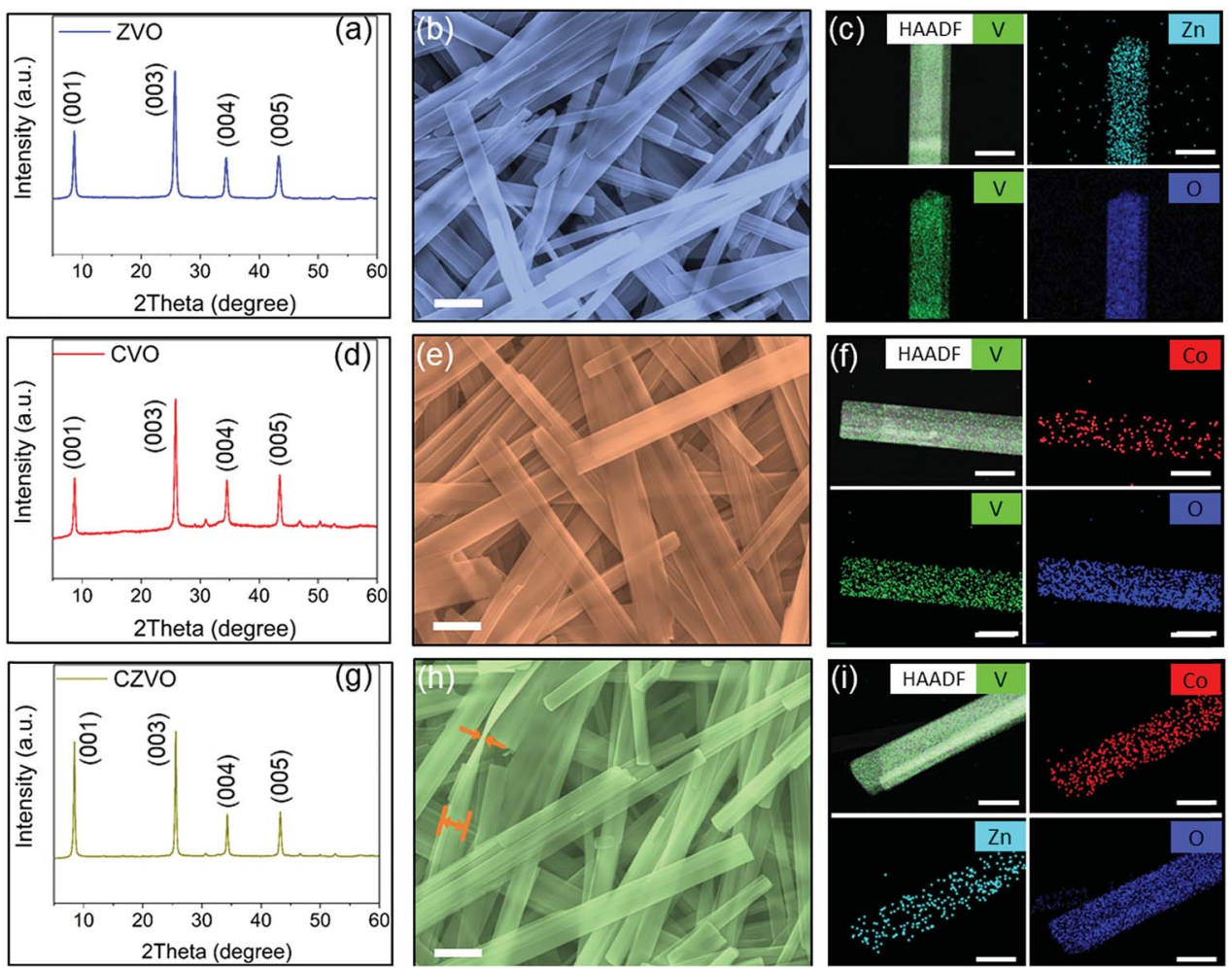

Fig. 1 (a) XRD patterns of ZVO, (d) CVO and (g) CZVO. (b) SEM images of ZVO, (e) CVO and (h) CZVO. Scale bar, $1 \mu \mathrm{m}$. (c) HAADF image with corresponding element distribution maps of ZVO, (f) CVO and (i) CZVO. Scale bar, $300 \mathrm{~nm}$. 
molar ratio is $1: 1$ (see Experimental section $\dagger$ ), energy dispersive spectroscopy (EDS) analyses using an atomic number sensitive high-angle annular dark-field detector (HAADF) indicates an atomic ratio of about $16: 9: 200$ for $\mathrm{Co}, \mathrm{Zn}$ and $\mathrm{V}$ in CZVO (Table S1 $\dagger$ ), pointing to a chemical formula of $\mathrm{Co}_{0.16}{ }^{-}$ $\mathrm{Zn}_{0.09} \mathrm{~V}_{2} \mathrm{O}_{5} \cdot n \mathrm{H}_{2} \mathrm{O}$. Obviously, CZVO with a Co to $\mathrm{Zn}$ ratio of 0.16 : 0.09 ratio forms preferentially. The EDS mapping patterns in Fig. 1c, $\mathrm{f}$ and i confirm the uniform distribution of $\mathrm{Zn}, \mathrm{V}, \mathrm{O}$ in ZVO, Co, V, O in CVO, and Co, Zn, V, O in CZVO, respectively. In addition, the surface areas of $\mathrm{ZVO}, \mathrm{CVO}$, and CZVO are determined to be $27.8,21.1,29.1 \mathrm{~m}^{2} \mathrm{~g}^{-1}$, respectively, through $\mathrm{N}_{2}$ gas sorption measurements (Fig. S5 $\dagger$ ). The pore size distribution obtained by a density functional theory (DFT) analysis reveals the coexistence of mesoporosity and macroporosity with pore sizes between 5 and $80 \mathrm{~nm}$ for all samples. The high-resolution Co $2 p$ XPS spectra of CVO and CZVO are presented in Fig. S6a and $b, \uparrow$ respectively. Satellite features are found for both samples at $\sim 787 \mathrm{eV}$, indicating a chemical state of $2+$ for Co. ${ }^{36}$ As shown in Fig. S6c and $d, \dagger$ the $\mathrm{Zn} 2 \mathrm{p}_{3 / 2}$ peaks of ZVO and CZVO appear at $\sim 1022 \mathrm{eV}$, which can be assigned to $\mathrm{Zn}^{2+} \cdot{ }^{37}$

The electrochemical properties were evaluated in half-cell configuration using sodium foil as both counter and reference electrode. Typical galvanostatic charge-discharge profiles for CVO, CZVO and ZVO at a low current density of $0.1 \mathrm{~A} \mathrm{~g}^{-1}$ are shown in Fig. 2a. CVO and CZVO deliver high initial discharge capacities of 563 and $434 \mathrm{~mA} \mathrm{~h} \mathrm{~g}{ }^{-1}$, respectively, while ZVO provides a lower value of $397 \mathrm{~mA} \mathrm{~h} \mathrm{~g}^{-1}$. The reversible initial charge capacities also follow the trend $\mathrm{CVO}>\mathrm{CZVO}>\mathrm{ZVO}$, with the Coulombic efficiencies reaching 64\% (CVO), 65\% (CZVO) and $60 \%$ (ZVO), respectively. The capacity loss can be attributed to irreversible side reactions, which lead to the formation of a solid-electrolyte interphase (SEI) on the electrode surface. ${ }^{\mathbf{3 8 - 4 1}}$ Fig. $2 \mathrm{~b}$ exhibits the rate performances of the three samples. At current rates ranging from $0.1-3 \mathrm{~A} \mathrm{~g}^{-1}$, CVO demonstrates the highest capacity. The high capacities obtained for CVO during the first several cycles at various current densities might be due to the lower atomic weight or to differences in electronic conductivity. Accordingly, CVO is expected to possess a higher electrical conductivity, resulting in higher charge storage capability. ${ }^{\mathbf{4 2 , 4 3}}$ The assumption is confirmed by impedance measurements. As shown in Fig. $\mathrm{S} 7, \dagger$ the electrical conductivity of CVO is determined to be $1.6 \times 10^{-6} \mathrm{~S} \mathrm{~cm}^{-1}$, which is slightly higher than $7.8 \times 10^{-7} \mathrm{~S} \mathrm{~cm}^{-1}$ and $1.4 \times 10^{-6} \mathrm{~S} \mathrm{~cm}^{-1}$ found for ZVO and CZVO, respectively. The long-term cycling tests of the samples are performed at a high current rate of $3 \mathrm{~A} \mathrm{~g}^{-1}$, starting with a pre-activation process, where the batteries were cycled at a low current density of $0.1 \mathrm{~A} \mathrm{~g}^{-1}$ for 5 cycles (Fig. 2c). The Coulombic efficiencies for the three samples reach values close to $100 \%$ after only a few cycles (Fig. S8†). The relatively low Coulombic efficiency during the first several cycles might be due to an unstable SEI, as described in other reports. ${ }^{44-46}$ During the first 500 cycles, CVO exhibits higher capacity than CZVO and $\mathrm{ZVO}$, in accordance with the results obtained at $0.1 \mathrm{~A} \mathrm{~g}^{-1}$ and the rate performance test. However, the next 500 cycles witness a dramatic capacity fading of CVO, while the capacities of CZVO and ZVO stay stable throughout the cycling test. After 1000 cycles of fast charging/discharging, CZVO is able to maintain a much higher capacity compared with ZVO and CVO. Specifically, a capacity of $85 \mathrm{~mA} \mathrm{~h} \mathrm{~g}^{-1}$ can be achieved for CZVO, which even exceeds its initial capacity of $73 \mathrm{~mA} \mathrm{~h} \mathrm{~g}^{-1}$. The reason for the capacity increase might be due to the improved wetting of the electrodes with the electrolyte during the first hundreds of cycles. ${ }^{47}$ Similar phenomenon was also observed for the other two samples. ZVO finally obtains a capacity of $38 \mathrm{~mA} \mathrm{~h}^{-1}$, with a capacity retention of nearly $97 \%$. For CVO only $27 \%$ of the initial capacity is maintained at the end of the cycling test. The
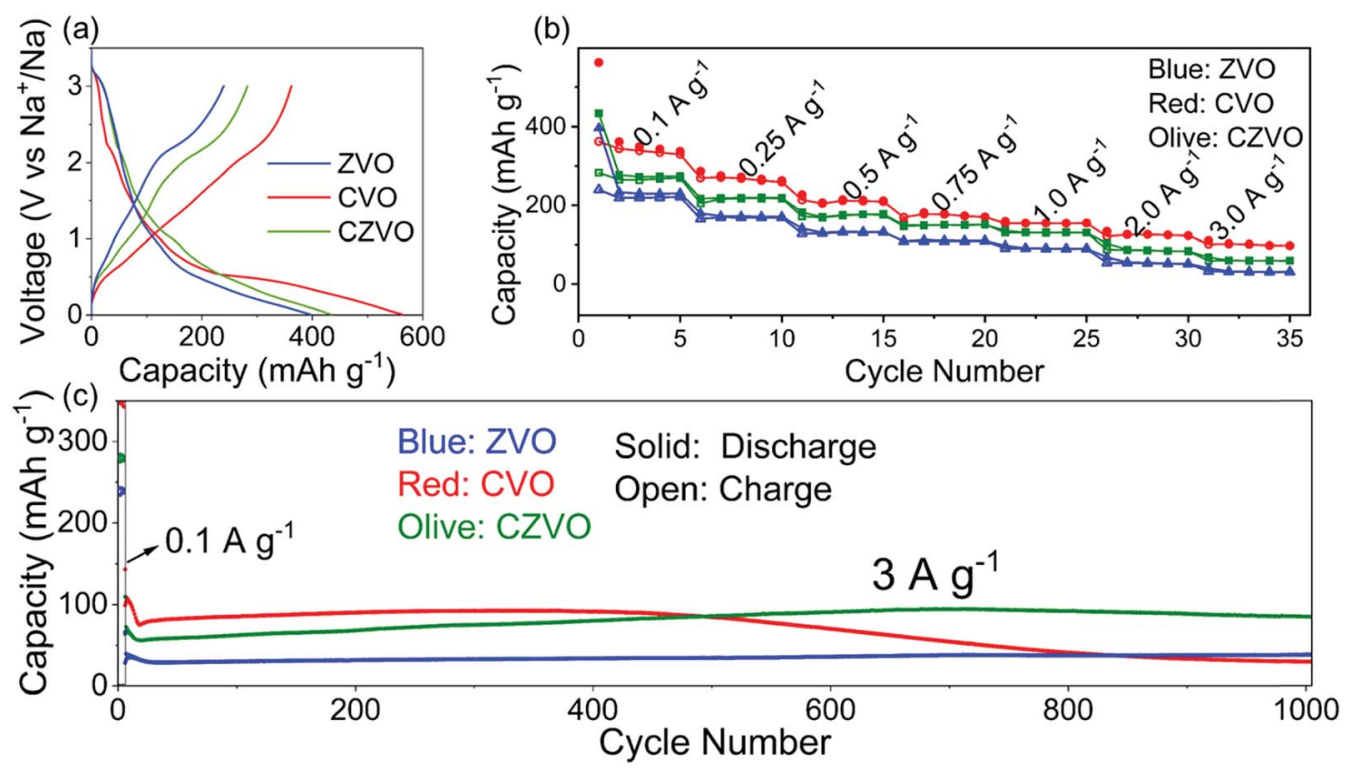

Fig. 2 (a) Galvanostatic charge-discharge profiles of ZVO, CVO and CZVO at $0.1 \mathrm{~A} \mathrm{~g}^{-1}$. (b) Comparison of the rate performance of ZVO, CVO, and CZVO. (c) Comparison of the long-term cycling performance of ZVO, CVO, and CZVO at a current density of $3 \mathrm{~A} \mathrm{~g}^{-1}$ after a pre-cycling procedure at $0.1 \mathrm{~A} \mathrm{~g}^{-1}$ for 5 cycles. 
results clearly indicate a much better structural stability of CZVO and ZVO during cycling compared with CVO.

The observed electrochemical behaviors during the cycling tests for the different samples may be attributed to the local electrostatic field that influences the intercalation of $\mathrm{Na}^{+}$ions. In order to gain insight into the underlying mechanisms at the atomistic scale, we carried out first-principles calculations based on the density functional theory (DFT) (for details see the Experimental section in the ESI $\dagger$ ). Because CZVO is nonstoichiometric and the $\mathrm{Zn}$ and Co interactions are dominant in the three samples, we concentrate on ZVO and CVO to reveal the influence of interlayer cations on the electrochemical performance. Fig. 3a presents the energy-minimized structures of ZVO and CVO. The formation of hydrogen bonds between water molecules coordinated to the $\mathrm{Zn}$ and Co ions and the oxygen atoms in the $\mathrm{VO}_{x}$ layers binds the layers to the $\mathrm{Zn} / \mathrm{Co}$ octahedra. As a result, as shown in the $2 \mathrm{D}$ electrostatic potential profile of the (002) plane, there are channel-like regions having low electrostatic potential (with blue color), whose width is comparable to the ionic diameter of $\mathrm{Na}^{+}(\sim 0.102 \mathrm{~nm})$. We therefore hypothesize that these channels serve as pathways for ion migration. Interestingly, by artificially inserting two $\mathrm{Na}^{+}$ into the unit cell in the simulations (Fig. 3b), CVO experiences a stress of $4.13 \mathrm{GPa}$, which is about $34 \%$ larger than that of ZVO (3.06 GPa), presumably due to a stronger electrostatic repulsion in CVO. We further compare the energy landscape by moving a $\mathrm{Na}^{+}$along the $(\overline{1} 10)$ direction (Fig. 3c), corresponding to the "channels" revealed in Fig. 3a. Clearly, the energy profiles share the same feature, with two energy barriers to be overcome, while following the same trend as revealed in Fig. $3 \mathrm{~b}$. The energy barrier in ZVO $(0.50 \mathrm{eV})$ is about $25 \%$ smaller than that in CVO $(0.67 \mathrm{eV})$. These features qualitatively capture the experimentally observed electrochemical performance, as the intercalation and diffusion of $\mathrm{Na}^{+}$are more energetically favorable in ZVO relative to CVO. Accordingly, we infer that the interlayer cations play a decisive role. In comparison to CVO, the interlayer $\mathrm{Zn}$ ions in CZVO do not only favor the Na-ion storage kinetics, but also the structural reversibility as confirmed by $e x$ situ XRD discussed below. Moreover, CZVO possesses higher electronic conductivity and larger interplanar spacing compared with ZVO, leading to a higher charge storage capability. All together, these effects might lead to the comparably high performance of CZVO among the studied metal vanadates.

Ex situ XRD patterns in Fig. 4 provide further information on the different Na-ion storage behaviors of the samples during cycling. For the ex situ XRD measurements, all three metal vanadates were cycled at $3 \mathrm{~A} \mathrm{~g}^{-1}$ for 500 cycles and 1000 cycles, respectively. The electrodes were taken out from the batteries, thoroughly rinsed with dimethyl carbonate and naturally dried at room temperature prior to the XRD measurements. As shown in Fig. 4a and c, intense (00l) diffraction patterns are observed for ZVO and CZVO even after 1000 cycles, indicating their high (a)

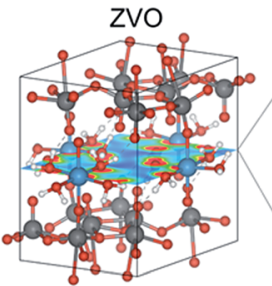

(b)

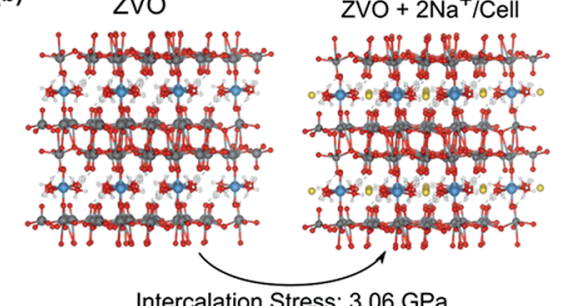

Electrostatic potential (002)
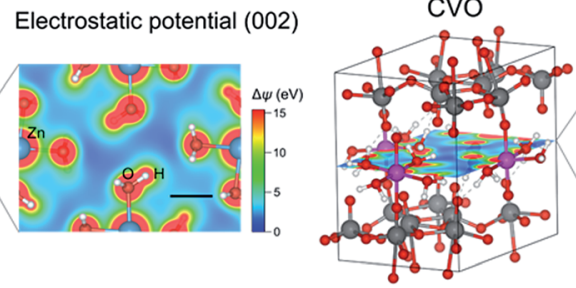

cVo

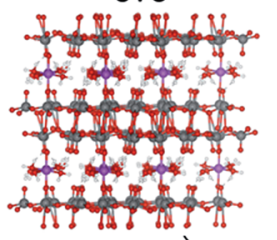

Intercalation Stress: $4.13 \mathrm{GPa}$

Electrostatic potential (002)

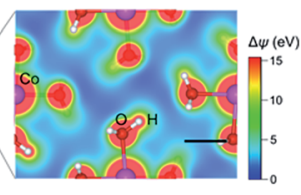

$\mathrm{CVO}+2 \mathrm{Na}^{+} / \mathrm{Cell}$

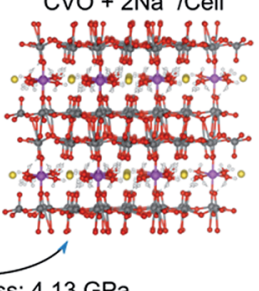

(c)
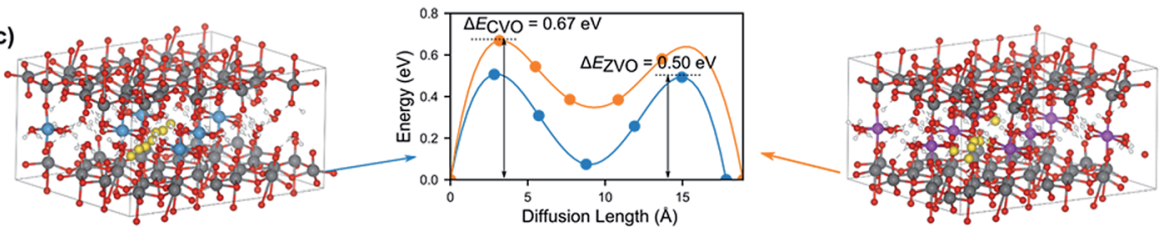

CVO $\mathrm{Na}^{+}$diffusion along $(\overline{1} 10)$

Fig. 3 (a) Electrostatic potential profiles of ZVO (left) and CVO (right) on the (002) plane from first principles simulations. The layered structure of transition metal vanadates creates low potential energy regions (blue color), which serve as preferred pathways for cations. Scale bars: $2 \AA$. (b) Stress and structural change of $\mathrm{Na}^{+}$intercalation from first principles simulations, with simulated structures $(2 \times 2 \times 2$ superlattice $)$ viewed along the (110) direction. ZVO shows smaller intercalation stress and volume change compared with CVO. (c) Simulated migration energy profiles of $\mathrm{Na}^{+}$along the (110) direction in ZVO (blue curve) and CVO (orange curve). The migration energy barrier in ZVO is $\sim 25 \%$ smaller than that in CVO. The simulation domain for each structure contains $2 \times 2 \times 1$ superlattice, while the migration is simulated within one unit cell. 

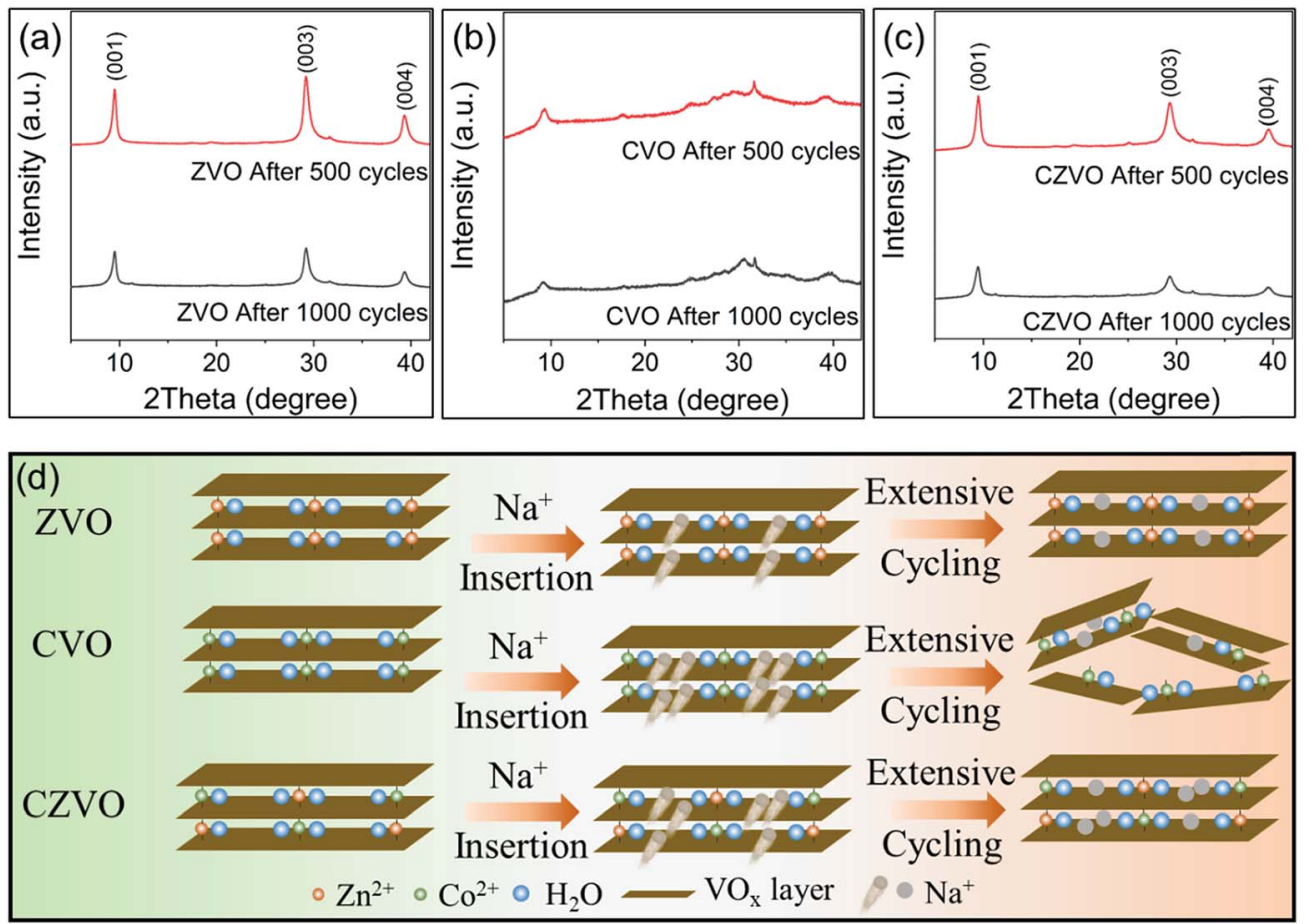

Fig. 4 Ex situ XRD patterns of the electrodes of (a) ZVO, (b) CVO and (c) CZVO after cycling at $3 \mathrm{~A} \mathrm{~g}^{-1}$ for 500 cycles and 1000 cycles, respectively. (d) Proposed schematic for the structural evolutions of ZVO, CZVO and CVO during cycling.

structural reversibility. In contrast, much broader and weaker diffraction peaks are found for CVO already after 500 cycles (Fig. $4 \mathrm{~b}$ ), pointing to a severe structural damage during the fast $\mathrm{Na}^{+}$insertion/extraction. The results provide direct evidence to explain the capacity fading of CVO starting from $\sim 500$ th cycle. The proposed structural evolutions of the three samples are depicted in Fig. 4d. The structural failure of CVO during cycling might be due to the joint effects of the large amount of initially inserted sodium ions, high structural stress during operation and sluggish Na-ion diffusion inside the structure, as endorsed by the first principles simulations. Ex situ SEM results are shown in Fig. S9. $\uparrow$ The belt-like morphology of ZVO and CZVO is preserved to some extent after 500 cycles. The morphology of $\mathrm{CVO}$, on the other hand, is severely affected after the cycling test, which provides further reason for the relatively low cycling stability of CVO compared with ZVO and CZVO. Furthermore, ex situ XPS results in Fig. S10† clearly show the Zn $2 p$ signals in the discharged ZVO and CZVO electrodes and Co 2p signals in the discharged CVO and CZVO electrodes, confirming that the interlayer cations remain in the structures after discharging.

Considering both specific capacity and cycling stability, CZVO exhibits the best performance. Thus, it is selected as a representative for further, more detailed investigations. Fig. $2 \mathrm{~b}$ displays the rate capability of CZVO. Reversible capacities of 296, 238, 196, 169, 148, 103 and $74 \mathrm{~mA} \mathrm{~h} \mathrm{~g}^{-1}$ were obtained at $0.1,0.25,0.5,0.75,1,2$ and $3 \mathrm{~A} \mathrm{~g}^{-1}$, respectively. Compared with some reported high-performance Na-ion insertion anode materials, CZVO exhibits a comparable high rate capability (Fig. S11†), ${ }^{48-54}$ indicating its great potential for high-rate Na-ion storage. In addition, the galvanostatic charge- discharge profiles of CZVO at $3 \mathrm{~A} \mathrm{~g}^{-1}$ (Fig. S12†) exhibit a nearly linear correlation between voltage and capacity, indicating a predominantly pseudocapacitive behavior during cycling, which will be discussed in detail below.

$\mathrm{CV}$ analysis is an efficient method to study the pseudocapacitance in electrode materials. ${ }^{55-58}$ In the CV data, the relation between the peak current and the sweep rate obeys a power law described as $i=a v^{b}$ (where $a$ and $b$ are appropriate parameters). The calculated $b$ value can be used to classify the current contribution from the capacitive effects and the diffusioncontrolled insertion processes..$^{55,57-59}$ Specifically, $b=0.5$ indicates a diffusion-controlled faradaic process, while at the other end $b=1$ represents a pseudocapacitive charge storage behavior, i.e., no diffusion limit. The $\mathrm{CV}$ curves of CZVO at sweep rates ranging from $0.1-1.0 \mathrm{mV} \mathrm{s}^{-1}$ are shown in Fig. S13. $\dagger$ As depicted in Fig. 5a, $b$ values for peak 1 and peak 2 are 0.87 and 0.78 , respectively. The results demonstrate the obvious pseudocapacitive effects in CZVO. The method can be further utilized to quantitatively separate the capacity contributed from the capacitive effects and the diffusion-controlled insertion processes through analyzing the dependence of the CV curves on the sweep rates. ${ }^{55}$ As shown in Fig. 5b, CZVO exhibits a very high capacitive contribution of $83.9 \%$ at a scan rate of $1 \mathrm{mV} \mathrm{s}^{-1}$. The relative contributions of capacitive currents at various sweep rates are shown in Fig. 5c, clearly showing the dominant pseudocapacitive effects in CZVO. While pseudocapacitance can result from large surface area materials like hollow or hierarchical structures, which provide many adsorption and desorption sites for sodium ions, ${ }^{60,61}$ in our case the surface areas are, according to $\mathrm{N}_{2}$ gas sorption measurements, relatively 


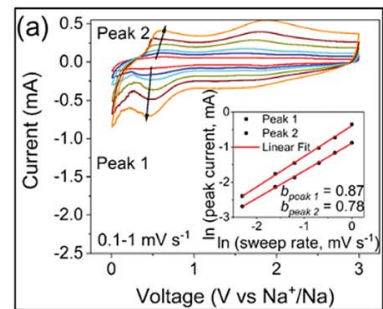

(d)

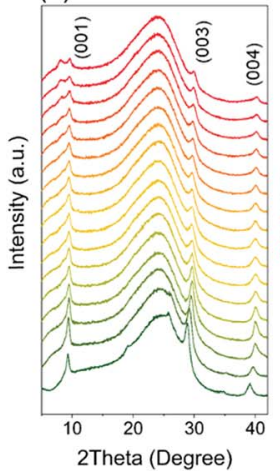

(e)

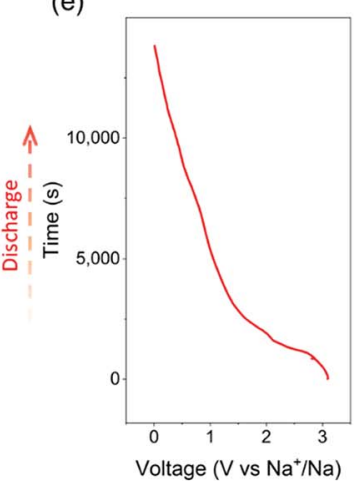

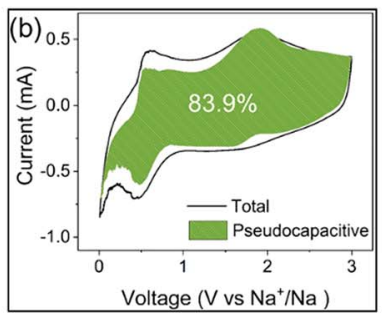

(f)

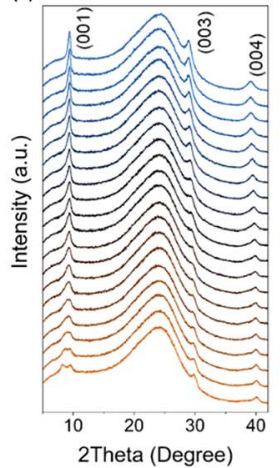

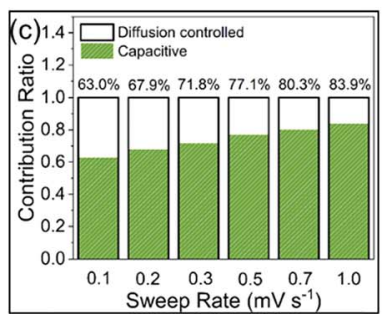

(g)

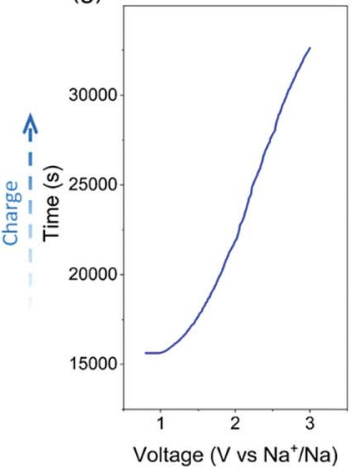

Fig. 5 (a) CV curves of CZVO at sweep rates from 0.1-1 $\mathrm{mV} \mathrm{s}^{-1}$. Inset: correlation between the peak current and the sweep rate. (b) Voltammetric response of CZVO at a sweep rate of $0.1 \mathrm{mV} \mathrm{s}^{-1}$. (c) Capacitive contributions of CZVO at various sweep rates $\left(0.1,0.2,0.3,0.5,0.7,1.0 \mathrm{mV} \mathrm{s}^{-1}\right.$ ). (d) Operando XRD patterns for CZVO during the first discharge and ( $f$ ) the first charge, and the corresponding voltage profiles for (e) the discharging and $(\mathrm{g})$ the charging processes.

low. Therefore, the reason for the high pseudocapacitive contribution in CZVO might be due to the fact that the charging/discharging processes are not limited by the Na-ion diffusion rate inside the structure, ${ }^{27,56}$ which can be attributed to the spacious interlayer channels enabling fast Na-ion storage.

Operando XRD measurements were applied to obtain deeper insight into the Na-ion storage mechanism of CZVO. A broad peak centered around $25^{\circ}$ is found for all patterns, which can be attributed to the operando cell as confirmed by the XRD pattern of the blank cell (Fig. S14 $\dagger$ ). At the starting stage of the operando XRD measurements, a mismatch between the operando and ex situ (Fig. 1g) diffraction patterns were observed, corresponding to a change of the $c$ lattice parameter from $10.39 \AA$ to $9.50 \AA$. This observation was also made for other vanadium-based energy storage materials possibly due to a partial loss of the loosely bound lattice water into the anhydrous electrolyte. ${ }^{27,34,62}$ The following discharging process witnesses a slight shift of the (001) peak from $9.3^{\circ}$ to $9.6^{\circ}$ (see Fig. 5d and e). The slight change corresponds to a decrease of the interlayer distance from $9.5 \AA$ to $9.2 \AA$, corresponding to a small volume change of only $3 \%$. Obviously, the spacious interlayer spacing in CZVO is able to provide fast Na-ion diffusion channels without significant volume change, which results in the stable cycling performance as discussed above. During discharge, a new diffraction peak appears at around $8^{\circ}$. Although this observation was made before for other reported vanadium-based alkaliion storage materials, the exact mechanism is still unknown. ${ }^{23,63,64}$ Finally, during charging, the structure follows the reverse evolution of discharge (see Fig. $5 \mathrm{f}$ and $\mathrm{g}$ ). In addition, the pattern obtained at fully charged state remains nearly unchanged compared with the initial pattern, suggesting the high structural reversibility of CZVO.

\section{Conclusions}

In summary, in this work we explored a series of metal vanadates (ZVO, CVO and CZVO) as high-rate Na-ion storage materials. Among the studied materials, CZVO exhibits the best cycling performance with $85 \mathrm{~mA} \mathrm{~h} \mathrm{~g}{ }^{-1}$, which remains after 1000 cycles at a high current rate of $3 \mathrm{~A} \mathrm{~g}^{-1}$. CV analysis reveals that CZVO possesses a dominant pseudocapacitance $(83.9 \%$ at $1 \mathrm{mV} \mathrm{s}^{-1}$ ) and operando XRD measurements demonstrate the small volume change of CZVO during Na-ion storage. The influence of the interlayer cations on the electrochemical behaviors of the metal vanadates was studied by DFT calculations, revealing that the interlayer $\mathrm{Zn}^{2+}$ has much lower electrostatic potential barrier than $\mathrm{Co}^{2+}$, which finally results in more favorable Na-ion diffusion in ZVO compared to CVO. Ex situ XRD measurements demonstrate the much higher structural stability of CZVO and ZVO compared with CVO during fast charging/discharging processes. Thus, the strong dependence of the electrochemical performance on the interlayer cations may open up completely new possibilities to enhance the performance of high-rate Na-ion storage or even rechargeable $\mathrm{Zn} / \mathrm{Mg} / \mathrm{Ca}$-ion storage through systematically tuning the interlayer cations of the layered electrode materials.

\section{Conflicts of interest}

There are no conflicts to declare. 


\section{Acknowledgements}

The authors are grateful to ETH Zurich (ETH Research Grant ETH-13 16-1) and the China Scholarship Council for financial support. T. Tian and C.-J. Shih are grateful for the financial support from ETH Zürich startup funding. L. Pan thanks the Office of China Postdoctoral Council for the fellowship (No. 32 Document of OCPC, 2017). We also thank D. Kundu for the help with the operando XRD measurements. We acknowledge the following colleagues for giving us access to their research equipment: G. Cossu and A. Rossi for XPS and A. Studart for SEM. We also thank the Scientific Center for Optical and Electron Microscopy (ScopeM) of ETH Zurich for providing the electron microscopy facilities.

\section{Notes and references}

1 N. Yabuuchi, K. Kubota, M. Dahbi and S. Komaba, Chem. Rev., 2014, 114, 11636-11682.

2 D. Kundu, E. Talaie, V. Duffort and L. F. Nazar, Angew. Chem., Int. Ed., 2015, 54, 3431-3448.

3 J. Cui, S. Yao and J.-K. Kim, Energy Storage Materials, 2017, 7, 64-114.

4 S. W. Kim, D. H. Seo, X. Ma, G. Ceder and K. Kang, Adv. Energy Mater., 2012, 2, 710-721.

5 W. Luo, F. Shen, C. Bommier, H. Zhu, X. Ji and L. Hu, Acc. Chem. Res., 2016, 49, 231-240.

6 H. Su, S. Jaffer and H. Yu, Energy Storage Materials, 2016, 5, 116-131.

7 S. Y. Hong, Y. Kim, Y. Park, A. Choi, N.-S. Choi and K. T. Lee, Energy Environ. Sci., 2013, 6, 2067-2081.

8 X. Geng, Y. Jiao, Y. Han, A. Mukhopadhyay, L. Yang and H. Zhu, Adv. Funct. Mater., 2017, 27, 1702998.

9 W. Luo, M. Allen, V. Raju and X. Ji, Adv. Energy Mater., 2014, 4, 1400554.

10 M. He, K. Kravchyk, M. Walter and M. V. Kovalenko, Nano Lett., 2014, 14, 1255-1262.

11 G.-L. Xu, Z. Chen, G.-M. Zhong, Y. Liu, Y. Yang, T. Ma, Y. Ren, X. Zuo, X.-H. Wu and X. Zhang, Nano Lett., 2016, 16, 3955-3965.

12 Y. Liu, N. Zhang, L. Jiao, Z. Tao and J. Chen, Adv. Funct. Mater., 2015, 25, 214-220.

13 X. Wang, Y. Chen, Y. Fang, J. Zhang, S. Gao and X. W. Lou, Angew. Chem., 2019, 131, 2701-2705.

14 F. Xie, L. Zhang, D. Su, M. Jaroniec and S. Z. Qiao, Adv. Mater., 2017, 29, 1700989.

15 Z. Liu, T. Lu, T. Song, X.-Y. Yu, X. W. Lou and U. Paik, Energy Environ. Sci., 2017, 10, 1576-1580.

16 Y. Li, C. Liu, Z. Xie, J. Yao and G. Cao, J. Mater. Chem. A, 2017, 5, 16590-16594.

17 Y. Fang, L. Xiao, X. Ai, Y. Cao and H. Yang, Adv. Mater., 2015, 27, 5895-5900.

18 Y. Fang, Q. Liu, L. Xiao, Y. Rong, Y. Liu, Z. Chen, X. Ai, Y. Cao, H. Yang and J. Xie, Chem, 2018, 4, 1167-1180.

19 N. A. Chernova, M. Roppolo, A. C. Dillon and M. S. Whittingham, J. Mater. Chem., 2009, 19, 2526-2552.
20 C. Zhang, H. Song, C. Liu, Y. Liu, C. Zhang, X. Nan and G. Cao, Adv. Funct. Mater., 2015, 25, 3497-3504.

21 Y. Zhu, L. Peng, D. Chen and G. Yu, Nano Lett., 2015, 16, 742747.

22 D. Kundu, B. D. Adams, V. Duffort, S. H. Vajargah and L. F. Nazar, Nat. Energy, 2016, 1, 16119.

23 P. Hu, T. Zhu, X. Wang, X. Wei, M. Yan, J. Li, W. Luo, W. Yang, W. Zhang and L. Zhou, Nano Lett., 2018, 18, 1758-1763.

24 P. He, M. Yan, G. Zhang, R. Sun, L. Chen, Q. An and L. Mai, Adv. Energy Mater., 2017, 7, 1601920.

25 Y. Cai, F. Liu, Z. Luo, G. Fang, J. Zhou, A. Pan and S. Liang, Energy Storage Materials, 2018, 13, 168-174.

26 Q. Wei, Q. Wang, Q. Li, Q. An, Y. Zhao, Z. Peng, Y. Jiang, S. Tan, M. Yan and L. Mai, Nano Energy, 2018, 47, 294-300.

27 H. Huang, D. Kundu, R. Yan, E. Tervoort, X. Chen, L. Pan, M. Oschatz, M. Antonietti and M. Niederberger, Adv. Energy Mater., 2018, 8, 1802800.

28 V. Augustyn, J. Come, M. A. Lowe, J. W. Kim, P.-L. Taberna, S. H. Tolbert, H. D. Abruña, P. Simon and B. Dunn, Nat. Mater., 2013, 12, 518.

29 R. Chen, M. Knapp, M. Yavuz, S. Ren, R. Witte, R. Heinzmann, H. Hahn, H. Ehrenberg and S. Indris, Phys. Chem. Chem. Phys., 2015, 17, 1482-1488.

30 K. J. Griffith, K. M. Wiaderek, G. Cibin, L. E. Marbella and C. P. Grey, Nature, 2018, 559, 556-563.

31 W. Kong, R. Gao, Q. Li, W. Yang, J.-B. Yang, L. Sun and X. Liu, J. Mater. Chem. A, 2019, 7, 9099-9109.

32 L. Li, H. Wang, W. Han, H. Guo, A. Hoser, Y. Chai and X. Liu, J. Electrochem. Soc., 2018, 165, A3854-A3861.

33 H. Wang, R. Gao, Z. Li, L. Sun, Z. Hu and X. Liu, Inorg. Chem., 2018, 57, 5249-5257.

34 Y. Oka, O. Tamada, T. Yao and N. Yamamoto, J. Solid State Chem., 1996, 126, 65-73.

35 Y. Oka, T. Yao and N. Yamamoto, J. Solid State Chem., 1999, 144, 181-187.

36 M. C. Biesinger, B. P. Payne, A. P. Grosvenor, L. W. Lau, A. R. Gerson and R. S. C. Smart, Appl. Surf. Sci., 2011, 257, 2717-2730.

37 M. C. Biesinger, L. W. Lau, A. R. Gerson and R. S. C. Smart, Appl. Surf. Sci., 2010, 257, 887-898.

38 S. Wenzel, T. Hara, J. Janek and A.-P. Environmental, Energy Environ. Sci., 2011, 4, 3342-3345.

39 S. Komaba, W. Murata, T. Ishikawa, N. Yabuuchi, T. Ozeki, T. Nakayama, A. Ogata, K. Gotoh and K. Fujiwara, Adv. Funct. Mater., 2011, 21, 3859-3867.

40 H. Wu, G. Chan, J. W. Choi, I. Ryu, Y. Yao, M. T. Mcdowell, S. W. Lee, A. Jackson, Y. Yang and L. Hu, Nat. Nanotechnol., 2012, 7, 310-315.

41 Y. Sun, L. Zhao, H. Pan, X. Lu, L. Gu, Y.-S. Hu, H. Li, M. Armand, Y. Ikuhara and L. Chen, Nat. Commun., 2013, 4, 1870.

42 B. Yan, M. Li, X. Li, Z. Bai, J. Yang, D. Xiong and D. Li, J. Mater. Chem. A, 2015, 3, 11773-11781.

43 L.-H. Hu, F.-Y. Wu, C.-T. Lin, A. N. Khlobystov and L.-J. Li, Nat. Commun., 2013, 4, 1687. 
44 K.-L. Hong, L. Qie, R. Zeng, Z.-Q. Yi, W. Zhang, D. Wang, W. Yin, C. Wu, Q.-J. Fan and W.-X. Zhang, J. Mater. Chem. A, 2014, 2, 12733-12738.

45 S. Wenzel, T. Hara, J. Janek and P. Adelhelm, Energy Environ. Sci., 2011, 4, 3342-3345.

46 J. Zhu, C. Chen, Y. Lu, Y. Ge, H. Jiang, K. Fu and X. Zhang, Carbon, 2015, 94, 189-195.

47 Z. Le, F. Liu, P. Nie, X. Li, X. Liu, Z. Bian, G. Chen, H. B. Wu and Y. Lu, ACS Nano, 2017, 11, 2952-2960.

48 H. Li, Y. Zhu, S. Dong, L. Shen, Z. Chen, X. Zhang and G. Yu, Chem. Mater., 2016, 28, 5753-5760.

49 X. Wang, S. Kajiyama, H. Iinuma, E. Hosono, S. Oro, I. Moriguchi, M. Okubo and A. Yamada, Nat. Commun., 2015, 6, 6544-6549.

50 H. Pan, X. Lu, X. Yu, Y. S. Hu, H. Li, X. Q. Yang and L. Chen, Adv. Energy Mater., 2013, 3, 1186-1194.

51 D. Su, S. Dou and G. Wang, Chem. Mater., 2015, 27, 60226029.

52 A. Ponrouch, A. Goñi and M. R. Palacín, Electrochem. Commun., 2013, 27, 85-88.

53 Y. Wen, K. He, Y. Zhu, F. Han, Y. Xu, I. Matsuda, Y. Ishii, J. Cumings and C. Wang, Nat. Commun., 2014, 5, ncomms5033.

54 Y. Fang, L. Xiao, J. Qian, Y. Cao, X. Ai, Y. Huang and H. Yang, Adv. Energy Mater., 2016, 6, 1502197.
55 J. Wang, J. Polleux, J. Lim and B. Dunn, J. Phys. Chem. C, 2007, 111, 14925-14931.

56 V. Augustyn, P. Simon and B. Dunn, Energy Environ. Sci., 2014, 7, 1597-1614.

57 E. Lim, C. Jo, M. S. Kim, M. H. Kim, J. Chun, H. Kim, J. Park, K. C. Roh, K. Kang and S. Yoon, Adv. Funct. Mater., 2016, 26, 3711-3719.

58 J. Liu, J. Wang, C. Xu, H. Jiang, C. Li, L. Zhang, J. Lin and Z. X. Shen, Adv. Sci., 2018, 5, 1700322.

59 H. Huang, X. Wang, E. Tervoort, G. Zeng, T. Liu, X. Chen, A. Sologubenko and M. Niederberger, ACS Nano, 2018, 12, 2753-2763.

60 Y. Fang, B. Y. Guan, D. Luan and X. W. Lou, Angew. Chem., Int. Ed., 2019, 58, 7739-7743.

61 D. Chao, C. Zhu, P. Yang, X. Xia, J. Liu, J. Wang, X. Fan, S. V. Savilov, J. Lin and H. J. Fan, Nat. Commun., 2016, 7, 12122.

62 S. Nordlinder, L. Nyholm, T. Gustafsson and K. Edström, Chem. Mater., 2006, 18, 495-503.

63 C. Cartier, A. Tranchant, M. Verdaguer, R. Messina and H. Dexpert, Electrochim. Acta, 1990, 35, 889-898.

64 C. Xia, J. Guo, P. Li, X. Zhang and H. N. Alshareef, Angew. Chem., Int. Ed., 2018, 57, 3943-3948. 